\title{
Domains of holomorphy for irreducible unitary representations of simple Lie groups
}

\section{Bernhard Krötz}

Max-Planck-Institut für Mathematik, Vivatsgasse 7, D-53111 Bonn, Germany

(e-mail: kroetz@mpim-bonn.mpg.de)

Oblatum 25-II-2007 \& 7-XI-2007

Published online: 5 December 2007 - (C) Springer-Verlag 2007

\section{Introduction}

Let us consider a unitary irreducible representation $(\pi, \mathscr{H})$ of a simple, non-compact and connected algebraic Lie group $G$. Let us denote by $K$ a maximal compact subgroup of $G$. According to Harish-Chandra, the Lie algebra submodule $\mathcal{H}_{K}$ of $K$-finite vectors of $\pi$ consists of analytic vectors for the representation, i.e. for all $v \in \mathscr{H}_{K}$ the orbit map

$$
f_{v}: G \rightarrow \mathcal{H}, g \mapsto \pi(g) v
$$

is real analytic. For these functions $f_{v}$ we determine, and in full generality, their natural domain of definition as holomorphic functions (see Theorem 5.1 below):

Theorem 1.1. Let $(\pi, \mathscr{H})$ be a unitary irreducible representation of $G$. Let $v \in \mathcal{H}$ be a non-zero $K$-finite vector and $f_{v}$ be the corresponding orbit map.

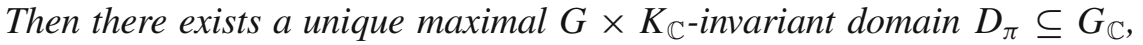
independent of $v$, to which $f_{v}$ extends holomorphically. Explicitly:

(i) $D_{\pi}=G_{\mathbb{C}}$ if $\pi$ is the trivial representation.

(ii) $D_{\pi}=\Xi^{+} K_{\mathbb{C}}$ if $G$ is Hermitian and $\pi$ is a non-trivial highest weight representation.

(iii) $D_{\pi}=\Xi^{-} K_{\mathbb{C}}$ if $G$ is Hermitian and $\pi$ is a non-trivial lowest weight representation.

(iv) $D_{\pi}=\Xi K_{\mathbb{C}}$ in all other cases.

In the theorem above $\Xi, \Xi^{+}, \Xi^{-}$are certain $G$-domains in $X_{\mathbb{C}}=G_{\mathbb{C}} / K_{\mathbb{C}}$ over $X=G / K$ with proper $G$-action. These domains are studied in this paper because of their relevance for the theorem above (see $[\mathrm{KO}]$ ). Let 
us mention that $\Xi$ is the familiar crown domain and that the inclusion $\Xi K_{\mathbb{C}} \subset D_{\pi}$ traces back to our joint work with Robert Stanton [KSI,KSII].

Acknowledgement: I am happy to point out that this paper is related to joint work with Eric M. Opdam [KO]. Also I would like to thank Joseph Bernstein who, over the years, helped me with his comments to understand the material much better.

Finally I appreciate the work of a very good referee who made many useful remarks on style and organization of the paper.

\section{Notation}

Throughout this paper $G$ shall denote a connected simple non-compact Lie group. We denote by $G_{\mathbb{C}}$ the universal complexification of $G$ and suppose:

- $G \subseteq G_{\mathbb{C}}$;

- $G_{\mathbb{C}}$ is simply connected.

We fix a maximal compact subgroup $K<G$ and form

$$
X=G / K,
$$

the associated Riemannian symmetric space of the non-compact type. The universal complexification $K_{\mathbb{C}}$ of $K$ will be realized as a subgroup of $G_{\mathbb{C}}$. We set

$$
X_{\mathbb{C}}=G_{\mathbb{C}} / K_{\mathbb{C}}
$$

and call $X_{\mathbb{C}}$ the affine complexification of $X$. Note that

$$
X \hookrightarrow X_{\mathbb{C}}, g K \mapsto g K_{\mathbb{C}}
$$

defines a $G$-equivariant embedding which realizes $X$ as a totally real form of the Stein symmetric space $X_{\mathbb{C}}$. We write $x_{0}=K_{\mathbb{C}} \in X_{\mathbb{C}}$ for the standard base point in $X_{\mathbb{C}}$.

However, the natural complexification of $X$ is not $X_{\mathbb{C}}$, but the crown domain $\Xi \subsetneq X_{\mathbb{C}}$ whose definition we recall now. We shall provide the standard definition of $\Xi$, see $[\mathrm{AG}]$.

Lie algebras of subgroups $L<G$ will be denoted by the corresponding lower case German letter, i.e. $\mathfrak{l}<\mathfrak{g}$; complexifications of Lie algebras are marked with a $\mathbb{C}$-subscript, i.e. $\mathfrak{l}_{\mathbb{C}}$ is the complexification of $\mathfrak{l}$.

Let us denote by $\mathfrak{p}$ the orthogonal complement to $\mathfrak{k}$ in $\mathfrak{g}$ with respect to the Cartan-Killing form. We set

$$
\hat{\Omega}=\{Y \in \mathfrak{p} \mid \operatorname{spec}(\operatorname{ad} Y) \subset(-\pi / 2, \pi / 2)\} .
$$

Then

$$
\Xi=G \exp (i \hat{\Omega}) \cdot x_{0} \subset X_{\mathbb{C}}
$$

is a $G$-invariant neighborhood of $X$ in $X_{\mathbb{C}}$, commonly referred to as crown domain. Sometimes it is useful to have an alternative, although less invariant 
picture of the crown domain: if $\mathfrak{a} \subset \mathfrak{p}$ is a maximal abelian subspace and $\Omega:=\hat{\Omega} \cap \mathfrak{p}$, then

$$
\Xi=G \exp (i \Omega) \cdot x_{0}
$$

The set $\Omega$ is nicely described through the restricted root system $\Sigma=\Sigma(\mathfrak{g}, \mathfrak{a})$ :

$$
\Omega=\{Y \in \mathfrak{a} \mid \alpha(Y)<\pi / 2 \forall \alpha \in \Sigma\} .
$$

If $\mathcal{W}$ is the Weyl group of $\Sigma$, then we note that $\Omega$ is $\mathcal{W}$-invariant.

Sometimes we will employ the root space decomposition $\mathfrak{g}=\mathfrak{a} \oplus \mathfrak{m} \oplus$ $\bigoplus_{\alpha \in \Sigma} \mathfrak{g}^{\alpha}$ with $\mathfrak{m}=\mathfrak{z}_{\mathfrak{k}}(\mathfrak{a})$ as usual. We choose a positive system $\Sigma^{+} \subset \Sigma$ and form the nilpotent subalgebra $\mathfrak{n}=\bigoplus_{\alpha \in \Sigma^{+}} \mathfrak{g}^{\alpha}$.

2.1. The example of $G=\operatorname{Sl}(2, \mathbb{R})$. For illustration and later use we will exemplify the above notions at the basic case of $G=\operatorname{Sl}(2, \mathbb{R})$.

We let $K=\mathrm{SO}(2, \mathbb{R})$ be our choice for the maximal compact subgroup and identify $X=G / K$ with the upper half plane $D^{+}:=\{z \in \mathbb{C} \mid \operatorname{Im} z>0\}$. We recall that

$$
X_{\mathbb{C}}=\mathbb{P}^{1}(\mathbb{C}) \times \mathbb{P}^{1}(\mathbb{C}) \backslash \operatorname{diag}\left[\mathbb{P}^{1}(\mathbb{C})\right]
$$

with $G_{\mathbb{C}}$ acting diagonally by fractional linear transformations. The $G$-embedding of $X=D^{+}$into $X_{\mathbb{C}}$ is given by

$$
z \mapsto(z, \bar{z}) \in X_{\mathbb{C}}
$$

If $D^{-}$denotes the lower half plane, then the crown domain is given by

$$
\Xi=D^{+} \times D^{-} \subseteq X_{\mathbb{C}}
$$

In addition we record two $G$-domains in $X_{\mathbb{C}}$ which sit above $\Xi$, namely:

$$
\begin{aligned}
& \Xi^{+}=D^{+} \times \mathbb{P}^{1}(\mathbb{C}) \backslash \operatorname{diag}\left[\mathbb{P}^{1}(\mathbb{C})\right], \\
& \Xi^{-}=\mathbb{P}^{1}(\mathbb{C}) \times D^{-} \backslash \operatorname{diag}\left[\mathbb{P}^{1}(\mathbb{C})\right] .
\end{aligned}
$$

Observe that $\Xi=\Xi^{+} \cap \Xi^{-}$.

\section{Remarks on $G$-invariant domains in $X_{\mathbb{C}}$ with proper action}

One defines elliptic elements in $X_{\mathbb{C}}$ by

$$
X_{\mathbb{C}, \mathrm{ell}}=G \exp (i \mathfrak{p}) \cdot x_{0}=G \exp (i \mathfrak{a}) \cdot x_{0} .
$$

The main result of [AG] was to show that $\Xi$ is a maximal domain in $X_{\mathbb{C} \text {,ell }}$ with $G$-action proper. In particular, $G$ acts properly on $\Xi$.

It was found in [KO] that $\Xi$ in general is not a maximal domain in $X_{\mathbb{C}}$ for proper $G$-action: the domains $\Xi^{+}$and $\Xi^{-}$from (2.2)-(2.3) yield 
counterexamples. To know all maximal domains is important for the theory of representations [KO, Sect. 4].

That $\Xi$ in general is not maximal for proper action is related to the unipotent model for the crown which was described in [KO]. To be more precise, we showed that there exists a domain $\hat{\Lambda} \subseteq \mathfrak{n}$ containing 0 such that

$$
\Xi=G \exp (i \hat{\Lambda}) \cdot x_{0} .
$$

Now there is a big difference between the unipotent parametrization (3.1) and the elliptic parametrization (2.1): If we enlarge $\Omega$ the result is no longer open; in particular, $X_{\mathbb{C} \text {,ell }}$ is not a domain. On the other hand, if we enlarge the open set $\hat{\Lambda}$ the resulting set is still open; in particular $X_{\mathbb{C}, \mathrm{u}}:=G \exp (i \mathfrak{n}) \cdot x_{0}$ is a domain. Thus, if there were a bigger domain than $\Xi$ with proper action, then it is likely by enlargement of $\hat{\Lambda}$.

We need some facts on the boundary of $\Xi$.

3.1. Boundary of $\Xi$. Let us denote by $\partial \Xi$ the topological boundary of $\Xi$ in $X_{\mathbb{C}}$. One shows that

$$
\partial_{\mathrm{ell}} \Xi:=G \exp (i \partial \Omega) \cdot x_{0} \subseteq \partial \Xi
$$

(cf. [KSII]) and calls $\partial_{\mathrm{ell}} \Xi$ the elliptic part of $\partial \Xi$. We define the unipotent part $\partial_{\mathrm{u}} \Xi$ of $\partial \Xi$ to be the complement to the elliptic part:

$$
\partial_{\mathrm{u}} \Xi=\partial \Xi \backslash \partial_{\mathrm{ell}} \boldsymbol{\Xi} .
$$

The relevance of $\partial_{\mathrm{u}} \Xi$ is as follows. Let $X \subset D \subseteq X_{\mathbb{C}}$ denote a $G$-domain with proper $G$-action. Then $D \cap \partial_{\text {ell }} \Xi=\emptyset$ by the above cited result of [AG]. Thus if $D \not \subset \Xi$, then one has

$$
D \cap \partial_{\mathrm{u}} \Xi \neq \varnothing .
$$

Let us describe $\partial_{\mathrm{u}} \Xi$ in more detail. For $Y \in \mathfrak{a}$ we define a reductive subalgebra of $\mathfrak{g}_{\mathbb{C}}$ by

$$
\mathfrak{g}_{\mathbb{C}}[Y]=\left\{Z \in \mathfrak{g}_{\mathbb{C}} \mid e^{-2 i \operatorname{ad}(Y)} \circ \sigma(Z)=Z\right\}
$$

with $\sigma$ the Cartan involution on $\mathfrak{g}_{\mathbb{C}}$ which fixes $\mathfrak{k}+i \mathfrak{p}$. Then there is a partial result on $\partial_{\mathrm{u}} \Xi$, for instance stated in $[\mathrm{FH}]$ :

$$
\begin{array}{r}
\partial_{\mathrm{u}} \Xi \subseteq\left\{G \exp (e) \exp (i Y) \cdot x_{0} \mid Y \in \partial \Omega,\right. \\
\left.0 \neq e \in \mathfrak{g}_{\mathbb{C}}[Y] \cap i \mathfrak{g} \text { nilpotent }\right\} .
\end{array}
$$

If $Y$ is such that only one root, say $\alpha$, attains the value $\pi / 2$, then we call $Y$ and as well the elements in the boundary orbit $G \exp (e) \exp (i Y) \cdot x_{0}$ regular. Accordingly we define the regular unipotent boundary $\partial_{\mathrm{u}, \mathrm{reg}} \Xi=$ $\left\{z \in \partial_{\mathrm{u}} \Xi \mid z\right.$ regular $\}$. Note that $\mathfrak{g}_{\mathbb{C}}[Y]$ is of especially simple form for 
regular $Y$, namely

$$
\mathfrak{g}_{\mathbb{C}}[Y]=i \mathfrak{a} \oplus \mathfrak{m} \oplus \mathfrak{g}[\alpha]^{-\theta} \oplus i \mathfrak{g}[\alpha]^{\theta}
$$

where $\mathfrak{g}[\alpha]=\mathfrak{g}^{\alpha} \oplus \mathfrak{g}^{-\alpha}$. Hence, in the regular situation, one can choose $e$ above to be in $i \mathfrak{g}[\alpha]^{\theta}+i \mathfrak{a}$. We summarize our discussion:

Proposition 3.1. Let $X \subset D \subseteq X_{\mathbb{C}}$ be a $G$-invariant domain with proper $G$-action which is not contained in $\Xi$. Then $D \cap \partial_{\mathrm{u}, \mathrm{reg}} \Xi \neq \emptyset$. More precisely, there exists $Y \in \partial \Omega$ regular (with $\alpha \in \Sigma$ the unique root attaining $\pi / 2$ on $Y$ ) and a non-zero nilpotent element $e \in i \mathfrak{g}[\alpha]^{\theta}+i \mathfrak{a}$ such that

$$
\exp (e) \exp (i Y) \cdot x_{0} \in \partial_{\mathrm{u}, \mathrm{reg}} \Xi \cap D .
$$

\section{Maximal domains for proper action}

The aim of this section is to classify all maximal $G$-domains in $X_{\mathbb{C}}$ which contain $X$ and maintain proper action. The answer will depend whether $G$ is of Hermitian type or not.

4.1. Non-Hermitian groups. The objective is to prove the following theorem:

Theorem 4.1. Suppose that $G$ is not of Hermitian type. If $X \subset D \subset X_{\mathbb{C}}$ is a $G$-invariant domain with proper $G$-action, then $D \subset \Xi$.

Before we can give the proof of the theorem some preparation is needed. The proof relies partly on a structural fact characterizing non-Hermitian groups (see Lemma 4.4 below) and on a precise knowledge of the basic case of $G=\operatorname{Sl}(2, \mathbb{R})$.

Let us begin with the relevant facts for $G=\operatorname{Sl}(2, \mathbb{R})$. With $E=\left(\begin{array}{ll}0 & 1 \\ 0 & 0\end{array}\right)$ and $T=\left(\begin{array}{cc}1 & 0 \\ 0 & -1\end{array}\right)$ our choices for $\mathfrak{a}$ and $\mathfrak{n}$ are

$$
\mathfrak{a}=\mathbb{R} \cdot T \quad \text { and } \quad \mathfrak{n}=\mathbb{R} \cdot E .
$$

Note that $\Omega=(-\pi / 4, \pi / 4) T$.

Then a slight modification of results in [KO, Sects. 3 and 4] yield:

Lemma 4.2. Let $G=\operatorname{Sl}(2, \mathbb{R})$ and $g \subset \mathbb{R}$ be an open subset. Then

$$
\Xi_{\mathfrak{g}}:=G \exp (i \mathcal{g} \cdot E) \cdot x_{0}
$$

is a $G$-invariant open subset of $X_{\mathbb{C}}$ and the following holds:

(i) $G$ does not act properly if $\{-1,1\} \subset \mathcal{g}$.

(ii) $\Xi=\Xi_{(-1,1)}$.

(iii) $\Xi^{+}=\Xi_{(-1, \infty)}$.

(iv) $\Xi^{-}=\Xi_{(-\infty, 1)}$. 
We also need that $\partial \Xi$ is a fiber bundle over the affine symmetric space $G / H$ where $H=\mathrm{SO}_{e}(1,1)$. Notice that $H$ is the stabilizer of the boundary point

$$
z_{H}:=\exp (-i \pi T / 4) \cdot x_{0}=(1,-1) \in \partial_{\mathrm{ell}} \Xi .
$$

Write $\tau$ for the involution on $G$, resp. $\mathfrak{g}$, fixing $H$, resp. $\mathfrak{h}$, and denote by $\mathfrak{g}=\mathfrak{h}+\mathfrak{q}$ the corresponding eigenspace decomposition. The $\mathfrak{h}$-module $\mathfrak{q}$ breaks into two eigenspaces $\mathfrak{q}=\mathfrak{q}^{+} \oplus \mathfrak{q}^{-}$with

$$
\mathfrak{q}^{ \pm}=\mathbb{R} \cdot e^{ \pm} \quad \text { where } \quad e^{ \pm}=\left(\begin{array}{cc}
1 & \mp 1 \\
\pm 1 & -1
\end{array}\right) .
$$

Finally write

$$
\mathcal{C}=\mathbb{R}_{\geq 0} \cdot e^{+} \cup \mathbb{R}_{\geq 0} \cdot e^{-}
$$

and $\mathcal{C}^{\times}=\mathcal{C} \backslash\{0\}$. Note that both $\mathcal{C}$ and $\mathcal{C}^{\times}$are $H$-stable. We cite [KO, Th. 3.1]:

Lemma 4.3. Let $G=\operatorname{Sl}(2, \mathbb{R})$. Then the map

$$
G \times{ }_{H} \mathcal{C} \rightarrow \partial \Xi, \quad[g, e] \mapsto g \exp (i e) \cdot z_{H}
$$

is a G-equivariant homeomorphism. Moreover,

(i) $\partial_{\mathrm{ell}} \Xi=G \cdot z_{H} \simeq G / H$,

(ii) $\partial_{\mathrm{u}} \Xi=G \exp \left(i \mathcal{C}^{\times}\right) \cdot z_{H} \simeq G \times{ }_{H} \mathcal{C}^{\times}$,

(iii) $\partial_{\mathrm{u}} \Xi=G \exp (i E) \cdot x_{0} \amalg G \exp (-i E) \cdot x_{0}$.

As a last piece of information we need a structural fact which is only valid for non-Hermitian groups.

Lemma 4.4. Suppose that $G$ is not of Hermitian type. Then for all $\alpha \in \Sigma$ and $E \in \mathfrak{g}^{\alpha}$ there exists an $m \in M=Z_{K}(\mathfrak{a})$ such that

$$
\operatorname{Ad}(m) E=-E .
$$

Proof. Let us remark first that we may assume that $G$ is of adjoint type. If $G$ is complex, then the assertion is clear as $T:=\exp (i \mathfrak{a}) \subset M$ provides us with the elements we are looking for. More generally for $\operatorname{dim} \mathfrak{g}^{\alpha}>1$ one knows (Kostant) that $M_{0}=\exp (\mathfrak{m})$ acts transitively on the unit sphere in $\mathfrak{g}^{\alpha}$ (cf. [Kos]).

In the sequel we use the terminology and tables of the classification of real simple Lie algebras as found in the monograph [K, App. C]. As $G$ is not Hermitian, Kostant's result leaves us with the following cases for $\mathfrak{g}$ : $\mathfrak{s l}(n, \mathbb{R})$ for $n \geq 3, \mathfrak{s o}(p, q)$ for $0,2 \neq p, q$ and $p+q>2, E I, E I I, E V$, $E V I, E V I I I, E I X, F I$ and $G$.

Now we make the following observation. The lemma is true for $G=$ $\mathrm{Sl}(3, \mathbb{R})$ as a simple matrix computation shows. Suppose that $\alpha$ is such that it can be put into an $A_{2}$-subsystem of $\Sigma$. As $\operatorname{dim} \mathfrak{g}^{\alpha}$ is one-dimensional (by 
our reduction) this means that we can put $E \in \mathfrak{g}^{\alpha}$ in a subalgebra isomorphic to $\mathfrak{s l}(3, \mathbb{R})$. Now it is important to recall the nature of the component group of $M$, see [K, Th. 7.55]. It follows that the $M$-group of $\mathrm{Sl}(3, \mathbb{R})$ (isomorphic to $\left.(\mathbb{Z} / 2 \mathbb{Z})^{2}\right)$ embeds into the $M$-group of $G$.

The $A_{2}$-reduction described above deletes most of the cases in our list. We remain with the orthogonal cases $\mathfrak{s o}(p, q)$ for $0,2 \neq p, q$ and $p \neq q$. A simple matrix computation, which we leave to the reader, finishes the proof.

Proof of Theorem 4.1. Suppose that $G$ is not of Hermitian type. Let $X \subset$ $D \subset \Xi$ be a $G$-invariant domain with proper $G$-action which is not contained in $\Xi$. We shall show that $D$ does not exist.

According to Proposition 3.1 we find a regular $Y \in \partial \Omega$ and a non-zero nilpotent $e \in \mathfrak{g}_{\mathbb{C}}[Y] \cap i \mathfrak{g}$ such that

$$
\exp (e) \exp (i Y) \cdot x_{0} \in \partial_{\mathrm{u}, \mathrm{reg}} \Xi \cap D .
$$

Let $\alpha \in \Sigma$ be the root corresponding to $Y$. Write $Y=Y^{\alpha}+Y^{\prime}$ with $Y^{\alpha}$, $Y^{\prime} \in \mathfrak{a}$ such that $\alpha\left(Y^{\prime}\right)=0$. It is known that $Y^{\alpha} \in \partial \Omega$ and $Y^{\prime} \in \Omega$. Hence we may use $\mathfrak{s l}(2)$-reduction which in conjunction with Lemma 4.3 implies the existence of $E^{\alpha} \in \mathfrak{g}^{\alpha}$ such that:

- $\left\{E^{\alpha}, \theta\left(E^{\alpha}\right),\left[E^{\alpha}, \theta\left(E^{\alpha}\right)\right]\right\}$ is an $\mathfrak{s l}(2)$-triple,

- $\exp \left(i E^{\alpha}\right) \exp \left(i Y^{\prime}\right) \cdot x_{0} \in \partial_{\mathrm{u}, \text { reg }} \Xi \cap D$.

Now, as $G$ is not of Hermitian type, Lemma 4.4 implies that there exists an element $m \in M$ such that $\operatorname{Ad}(m) E^{\alpha}=-E^{\alpha}$. Hence

$$
\exp \left(-i E^{\alpha}\right) \exp \left(i Y^{\prime}\right) \cdot x_{0} \in \partial_{\mathrm{u}, \text { reg }} \Xi
$$

as well. But this contradicts Lemma 4.2(i).

4.2. Hermitian groups. Let now $G$ be of Hermitian type and $G \subseteq P^{-} K_{\mathbb{C}} P^{+}$ be a Harish-Chandra decomposition of $G$ in $G_{\mathbb{C}}$. We define flag varieties

$$
F^{+}=G_{\mathbb{C}} / K_{\mathbb{C}} P^{+} \quad \text { and } \quad F^{-}=G_{\mathbb{C}} / K_{\mathbb{C}} P^{-}
$$

and inside of them we declare the flag domains

$$
D^{+}=G K_{\mathbb{C}} P^{+} / K_{\mathbb{C}} P^{+} \quad \text { and } \quad D^{-}=G K_{\mathbb{C}} P^{-} / K_{\mathbb{C}} P^{-} .
$$

Then in the

$$
X_{\mathbb{C}} \hookrightarrow F^{+} \times F^{-}, \quad g K_{\mathbb{C}} \mapsto\left(g K_{\mathbb{C}} P^{+}, g K_{\mathbb{C}} P^{-}\right)
$$

identifies $X_{\mathbb{C}}$ as a Zariski open affine piece of $F^{+} \times F^{-}$. In more detail: As $G$ is of Hermitian type, there exist $w_{0} \in N_{G_{\mathbb{C}}}\left(K_{\mathbb{C}}\right)$ such that $w_{0} P^{ \pm} w_{0}^{-1}=P^{\mp}$. 
In turn, this element induces a $G_{\mathbb{C}}$-equivariant biholomorphic map:

$$
\phi: F^{+} \rightarrow F^{-}, g K_{\mathbb{C}} P^{+} \mapsto g w_{0} K_{\mathbb{C}} P^{-} .
$$

With that the embedding (4.1) gives the following identification of $X_{\mathbb{C}}$ :

$$
X_{\mathbb{C}}=\left\{(z, w) \in F^{+} \times F^{-} \mid \phi(z) \mathrm{T} w\right\},
$$

where $T$ stands for the transversality notion in the flag variety $F^{-}$. We recall what it means to be transversal. First note that the notion is $G_{\mathbb{C}}$-invariant, i.e. for $z, w \in F^{-}$and $g \in G_{\mathbb{C}}$ one has $z \top w$ if and only if $g z \top g w$. Now for the base point $z^{-}=K_{\mathbb{C}} P^{-} \in F^{-}$one has $z^{-} \mathrm{T} w$ if and only if $w \in P^{-} w_{0} z^{-}$.

We keep the realization of $X_{\mathbb{C}}$ in $F^{+} \times F^{-}$(cf. (4.1)) in mind and recall the description of $\Xi$ :

$$
\Xi=D^{+} \times D^{-}
$$

(see $[\mathrm{KSII}])$.

For subsets $X^{ \pm} \subset F^{ \pm}$we write $X^{+} \times_{\top} X^{-}$for those elements $\left(x^{+}, x^{-}\right) \in$ $X^{+} \times X^{-}$which are transversal, i.e. $\phi\left(x^{+}\right) \top x^{-}$. With this terminology in mind we finally define

$$
\begin{aligned}
& \Xi^{+}=D^{+} \times_{\mathrm{T}} F^{-}, \\
& \Xi^{-}=F^{+} \times_{\mathrm{T}} D^{-} .
\end{aligned}
$$

4.2.1. Basic structure theory of $\Xi^{+}$and $\Xi^{-}$. It is obvious that both $\Xi^{+}$ and $\Xi^{-}$are open and $G$-invariant. However, as was pointed out by the referee, it is a priori not clear that they are connected. In order to see this let $p_{+}: \Xi^{+} \rightarrow D^{+}$be the projection onto the first factor. Likewise we define $p_{-}: \Xi^{-} \rightarrow D^{-}$.

Proposition 4.5. Let $\epsilon \in\{-,+\}$. The map $p_{\epsilon}: \Xi^{\epsilon} \rightarrow D^{\epsilon}$ induces the structure of a holomorphic fiber bundle with fiber isomorphic to $P^{\epsilon}$.

Proof. We confine ourselves with the case $\epsilon=+$.

As $p_{+}$is $G$-equivariant and $D^{+}$is $G$-homogeneous, it is sufficient to determine the fiber $p_{+}^{-1}\left(z^{+}\right)$. Recall that $z^{+}=K_{\mathbb{C}} P^{+} \in F^{+}$is the base point. Now

$$
p_{+}^{-1}\left(z^{+}\right)=\left\{\left(z^{+}, w\right) \in F^{+} \times F^{-} \mid \phi\left(z^{+}\right) \top w\right\} .
$$

Observe that $\phi\left(z^{+}\right)=w_{0} z^{-}$and that $w_{0} z^{-} \mathrm{T} w$ is equivalent to $z^{-} \mathrm{\top} w_{0}^{-1} w$. By the definition of transversality this means that $w_{0}^{-1} w \in P^{-} w_{0} z^{-}$or $w \in w_{0} P^{-} w_{0} z^{-}$. It is no loss of generality to assume that $w_{0}=w_{0}^{-1}$. So we arrive at $w \in P^{+} z^{-}$and this concludes the proof of the proposition.

Corollary 4.6. Both $\Xi^{+}$and $\Xi^{-}$are contractible.

It was observed by the the referee that Proposition 4.5 allows the following interesting reformulation. 
Corollary 4.7. The map

$$
G \times{ }_{K} P^{+} \rightarrow \Xi^{+}, \quad[g, p] \mapsto\left(g z^{+}, g p z^{-}\right)
$$

is a G-equivariant diffeomorphism. In particular $\Xi^{+}$is G-biholomorphic to $T^{0,1} D^{+}$, the antiholomorphic tangent bundle of $D^{+}$. Likewise, $\Xi^{-}$is G-biholomorphic to $T^{0,1} D^{-}$.

Corollary 4.7 combined with the Harish-Chandra decomposition implies that $\Xi^{\epsilon} \simeq D^{\epsilon} \times P^{\epsilon}$ as complex manifolds. In particular $\Xi^{\epsilon}$ is Stein.

The fact that $K_{\mathbb{C}}$ normalizes $P^{\epsilon}$ allows us to speak of $G \times P^{\epsilon}$-invariant domains in $X_{\mathbb{C}}$. It follows from (4.1) and Corollary 4.7 that $\Xi^{\epsilon}$ is $G \times P^{\epsilon}$ invariant.

Proposition 4.8. Let $\epsilon \in\{-,+\}$. The real group $G$ acts properly on $\Xi^{\epsilon}$. Moreover $\Xi^{\epsilon}$ is a maximal $G \times P^{\epsilon}$-invariant domain in $X_{\mathbb{C}}$ for proper G-action.

Proof. As the $G$-action is proper on $D^{\epsilon}$, it follows that $G$ acts properly on $\Xi^{\epsilon}$. In the sequel we deal with $\epsilon=+$ only. It remains to show that $\Xi^{+}$is a maximal $G \times P^{+}$-invariant domain in $X_{\mathbb{C}}$ for proper $G$-action. We argue by contradiction and suppose that $D \supsetneq \Xi^{+}$is a $G \times P^{+}$domain in $X_{\mathbb{C}}$ with proper $G$-action. Then $D=\left(D_{0} \times F^{-}\right) \cap X_{\mathbb{C}}$ with $D_{0} \supsetneq D^{+}$a $G$-domain with proper action. Now recall the following facts:

- There are only finitely many $G$-orbits in $F^{+}$.

- There are precisely two orbits with proper $G$-action: $D^{+}$and $\phi^{-1}\left(D^{-}\right)$.

The assertion follows.

Remark 4.9. Suppose that $G$ is of Hermitian type. Then it can be shown that if $X \subseteq D \subseteq X_{\mathbb{C}}$ is a $G$-invariant domain with proper $G$-action, then $D \subseteq \Xi^{+}$or $D \subseteq \Xi^{-}$.

As we will not need this fact, we refrain from a proof.

If $D \subseteq X_{\mathbb{C}}$ is a subset, then we write $D K_{\mathbb{C}}$ for its preimage in $G_{\mathbb{C}}$ under the canonical projection $G_{\mathbb{C}} \rightarrow X_{\mathbb{C}}$.

Proposition 4.10. The following assertions hold:

(i) $\Xi^{+} K_{\mathbb{C}}=G K_{\mathbb{C}} P^{+}$,

(ii) $\Xi^{-} K_{\mathbb{C}}=G K_{\mathbb{C}} P^{-}$.

Proof. It suffices to prove (i). Recall the embedding (4.1), and the definition of transversality condition. We deduce that $P^{+} \subset \Xi^{+} K_{\mathbb{C}}$. As $\Xi^{+} K_{\mathbb{C}}$ is $G \times K_{\mathbb{C}}$-invariant, it follows that $G P^{+} K_{\mathbb{C}}=G K_{\mathbb{C}} P^{+} \subset \Xi^{+} K_{\mathbb{C}}$.

Conversely, Corollary 4.7 implies that $G P^{+}$maps onto $\Xi^{+}$and thus $\Xi^{+} \subset G P^{+} K_{\mathbb{C}}$. 
We conclude this subsection with some easy facts on the structure of $\Xi^{+}$ and $\Xi^{-}$which will be used later on.

4.2.2. Unipotent model for $\Xi^{+}$and $\Xi^{-}$. We begin with the unipotent parameterization of $\Xi^{+}$and $\Xi^{-}$. Some terminology is needed.

According to $\mathrm{C}$. Moore, $\Sigma$ is of type $C_{n}$ or $B C_{n}$. Hence we find a subset $\left\{\gamma_{1}, \ldots, \gamma_{n}\right\}$ of long strongly orthogonal restricted roots. We fix $E_{j} \in \mathfrak{g}^{\gamma_{j}}$ such that $\left\{E_{j}, \theta\left(E_{j}\right),\left[E_{j}, \theta E_{j}\right]\right\}$ becomes an $\mathfrak{s l}(2)$-triple. Set $T_{j}:=1 / 2\left[E_{j}, \theta E_{j}\right]$ and note that

$$
\Omega=\bigoplus_{j=1}^{n}(-\pi / 2, \pi / 2) T_{j}
$$

We set $V=\bigoplus_{j=1}^{n} \mathbb{R} \cdot E_{j}$ and take a cube inside $V$ by

$$
\Lambda=\bigoplus_{j=1}^{n}(-1,1) E_{j}
$$

In $[\mathrm{KO}$, Sect. 8], we have shown that

$$
\Xi=G \exp (i \Lambda) \cdot x_{0} .
$$

In this parametrization of $\Xi$ the unipotent boundary piece has a simple description:

$$
\partial_{\mathrm{u}} \Xi=G \exp (i \partial \Lambda) \cdot x_{0} .
$$

The strategy now is to enlarge $\Xi$ by enlarging $\Lambda$ while maintaining that the object stays a domain on which $G$ acts properly. But now we have to be a little bit careful with our choice of $E_{j}$. Replacing $E_{j}$ by $-E_{j}$ has no effect for the matters cited above, but for the sequel. Our choice is such that $\gamma_{1}, \ldots, \gamma_{n}$ are positive roots (this determines the non-compact roots in $\Sigma^{+}$ uniquely). We set

$$
\Lambda^{+}=\bigoplus_{j=1}^{n}(-1, \infty) E_{j} \quad \text { and } \quad \Lambda^{-}=\bigoplus_{j=1}^{n}(-\infty, 1) E_{j} .
$$

Then, a direct generalization of Lemma 4.2(iii), (iv) yields:

Proposition 4.11. The following assertions hold:

(i) $\Xi^{+}=G \exp \left(i \Lambda^{+}\right) \cdot x_{0}$,

(ii) $\Xi^{-}=G \exp \left(i \Lambda^{-}\right) \cdot x_{0}$.

Remark 4.12. If we define subcones of the nilcone $\mathcal{N} \subseteq \mathfrak{g}$ by

$$
\mathcal{N}^{+}=\operatorname{Ad}(K)\left[\bigoplus_{j=1}^{n}[0, \infty) E_{j}\right] \quad \text { and } \quad \mathcal{N}^{-}=-\mathcal{N}^{+},
$$


then one can show that the maps

$$
G \times_{K} \mathcal{N}^{ \pm} \rightarrow \Xi^{ \pm}, \quad[g, Y] \mapsto g \exp (i Y) \cdot x_{0}
$$

are homeomorphic.

\section{Representation theory}

Let $(\pi, \mathscr{H})$ be a unitary representation of $G$ and $\mathscr{H}_{K}$ the underlying HarishChandra module of $K$-finite vectors. Notice that $\mathscr{H}_{K}$ is naturally a module for $K_{\mathbb{C}}$.

We say that $(\pi, \mathscr{H})$ is a highest, resp. lowest, weight representation if $G$ is of Hermitian type and $\mathfrak{p}^{+}=\operatorname{Lie}\left(P^{+}\right)$, resp. $\mathfrak{p}^{-}$, acts on $\mathscr{H}_{K}$ in a finite manner.

We turn to the main result of this paper.

Theorem 5.1. Let $(\pi, \mathscr{H})$ be a unitary irreducible representation of $G$. Let $v \in \mathcal{H}$ be a non-zero $K$-finite vector and

$$
f_{v}: G \rightarrow \mathcal{H}, \quad g \mapsto \pi(g) v
$$

the corresponding orbit map. Then there exists a unique maximal $G \times K_{\mathbb{C}-}$ invariant domain $D_{\pi} \subseteq G_{\mathbb{C}}$, independent of $v$, to which $f_{v}$ extends holomorphically. Explicitly:

(i) $D_{\pi}=G_{\mathbb{C}}$ if $\pi$ is the trivial representation.

(ii) $D_{\pi}=\Xi^{+} K_{\mathbb{C}}$ if $G$ is Hermitian and $\pi$ is a non-trivial highest weight representation.

(iii) $D_{\pi}=\Xi^{-} K_{\mathbb{C}}$ if $G$ is Hermitian and $\pi$ is a non-trivial lowest weight representation.

(iv) $D_{\pi}=\Xi K_{\mathbb{C}}$ in all other cases.

Proof. If $\pi$ is trivial, then the assertion is clear. So let us assume that $\pi$ is non-trivial in the sequel. Fix a nonzero $K$-finite vector $v$ and consider the orbit map $f_{v}: G \rightarrow \mathcal{H}$. We recall the following two facts:

- $f_{v}$ extends to a holomorphic $G$-equivariant map $f_{v}: \Xi K_{\mathbb{C}} \rightarrow \mathscr{H}$ (see [KSII, Th. 1.1]).

- If $D_{v} \subseteq G_{\mathbb{C}}$ is a $G \times K_{\mathbb{C}}$-invariant domain to which $f_{v}$ extends holomorphically, then $G$ acts properly on $D_{v} / K_{\mathbb{C}}$ (see [KO, Th. 4.3]).

We begin with the case where $G$ is not of Hermitian type. Here the assertion follows from the bulleted items above in conjunction with Theorem 4.1.

So we may assume for the remainder that $G$ is of Hermitian type. If $\pi$ is a highest weight representation, then it is clear that $f_{v}$ extends to a holomorphic map $G K_{\mathbb{C}} P^{+} \rightarrow \mathcal{H}$. Thus, in this case $\Xi^{+} K_{\mathbb{C}}=G K_{\mathbb{C}} P^{+}$(cf. Proposition 4.10 ) is a maximal domain of definition for $f_{v}$ by Proposition 4.8 and the second bulleted item from above. Likewise, if $(\pi, \mathscr{H})$ is 
a lowest weight representation, then $\Xi^{-} K_{\mathbb{C}}$ is a maximal domain of definition of $f_{v}$. As both $\Xi^{+}$and $\Xi^{-}$are simply connected with sufficiently regular boundary, it follows that these maximal domains are in fact unique.

It remains to show:

- If $f_{v}$ extends holomorphically on a domain $D \supset \Xi$ such that $D \cap$ $\left[\Xi^{+} \backslash \Xi\right] \neq \varnothing$, then $(\pi, \mathscr{H})$ is a highest weight representation.

- If $f_{v}$ extends holomorphically $\mathrm{p}$ on a domain $D \supset \Xi$ such that $D \cap$ $\left[\Xi^{-} \backslash \Xi\right] \neq \emptyset$, then $(\pi, \mathscr{H})$ is a lowest weight representation.

It is sufficient to deal with the first case. So suppose that $f_{v}$ extends to a bigger domain $D$ such that $D \cap\left[\Xi^{+} \backslash \Xi\right] \neq \emptyset$. Taking derivatives and applying the fact that $d \pi\left(\mathcal{U}\left(\mathfrak{g}_{\mathbb{C}}\right)\right) v=\mathscr{H}_{K}$, we see that $f_{u}$ extends to $D$ for all $u \in \mathscr{H}_{K}$. By Proposition 3.1, (4.3) and our assumption we find $1 \leq j \leq n$ be such that $\exp \left(i E_{j}\right) \exp (i Y) \cdot x_{0} \in D$ for some $Y \in \Omega$ with $\gamma_{j}(Y)=0$. Let $G_{j}<G$ be the analytic subgroup corresponding to the $\mathfrak{s l}(2)$-triple $\left\{E_{j}, \theta\left(E_{j}\right),\left[E_{j}, \theta\left(E_{j}\right)\right]\right\}$. Basic representation theory of type I-groups in conjunction with [KO, Th. 4.7], yields that $\left.\pi\right|_{G_{j}}$ breaks into a direct sum of highest weight representations. Applying $N_{K}(\mathfrak{a})$ (which in particular permutes the $G_{k}$ and preserves $\mathscr{H}_{K}$ ) we see that above matters hold for any other $G_{k}$ as well (note that $Y$ might change but this does not matter as $\Omega$ is $N_{K}(\mathfrak{a})$-invariant). It follows that $\pi$ is a highest weight representation and completes the proof of the theorem.

Remark 5.2. The domains $\Xi, \Xi^{+}$and $\Xi^{-}$are independent of the choice of the connected group $G$. Accordingly, the above theorem holds for all simple connected non-compact Lie groups $G$, i.e. we can drop the assumption that $G \subseteq G_{\mathbb{C}}$ and $G_{\mathbb{C}}$ simply connected.

Problem 5.3. The above theorem should hold true for all irreducible admissible Banach representations of $G$ under the reservation that (i) gets modified to: $D_{\pi}=G_{\mathbb{C}}$ if $\pi$ is finite dimensional.

\section{References}

[AG] Akhiezer, D.N., Gindikin, S.G.: On Stein extensions of real symmetric spaces. Math. Ann. 286, 1-12 (1990)

[FH] Fels, G., Huckleberry, A.: Characterization of cycle domains via Kobayashi hyperbolicity. Bull. Soc. Math. Fr. 133(1), 121-144 (2005)

[K] Knapp, A.W.: Lie Groups Beyond an Introduction, 2nd edn. Prog. Math., vol. 140. Birkhäuser, Boston (2002)

[Kos] Kostant, B.: A branching law for subgroups fixed by an involution and a noncompact analogue of the Borel-Weil theorem. In: Noncommutative Harmonic Analysis, Prog. Math., vol. 220, pp. 291-353. Birkhäuser, Boston (2004)

[KO] Krötz, B., Opdam, E.M.: Analysis on the crown domain. Geom. Funct. Anal. (to appear), math.RT/0606213

[KSI] Krötz, B., Stanton, R.: Holomorphic extensions of representations: (I) automorphic functions. Ann. Math. 159, 641-724 (2004)

[KSII] Krötz, B., Stanton, R.: Holomorphic extensions of representations: (II) geometry and harmonic analysis. Geom. Funct. Anal. 15, 190-245 (2005) 